\title{
Bombs and Flares at the Surface and Lower Atmosphere of the Sun
}

\author{
V. H. Hansteen ${ }^{1}$, V. Archontis ${ }^{2}$, T. M. D. Pereira ${ }^{1}$, M. Carlsson ${ }^{1}$, L. Rouppe van der Voort ${ }^{1}$, and J. Leenaarts ${ }^{3}$ \\ ${ }^{1}$ Institute of Theoretical Astrophysics, University of Oslo, Norway, PB 1029 Blindern, NO-0315 Oslo, Norway \\ ${ }^{2}$ School of Mathematics and Statistics, St. Andrews University, St. Andrews, KY169SS, UK \\ ${ }^{3}$ Institute for Solar Physics, Dept. of Astronomy, Stockholm University, Roslagstullbacken 21 SE-10691 Stockholm, Sweden \\ Received 2017 February 1; revised 2017 March 16; accepted 2017 March 16; published 2017 April 10
}

\begin{abstract}
A spectacular manifestation of solar activity is the appearance of transient brightenings in the far wings of the H $\alpha$ line, known as Ellerman bombs (EBs). Recent observations obtained by the Interface Region Imaging Spectrograph have revealed another type of plasma "bombs" (UV bursts) with high temperatures of perhaps up to $8 \times 10^{4} \mathrm{~K}$ within the cooler lower solar atmosphere. Realistic numerical modeling showing such events is needed to explain their nature. Here, we report on 3D radiative magnetohydrodynamic simulations of magnetic flux emergence in the solar atmosphere. We find that ubiquitous reconnection between emerging bipolar magnetic fields can trigger EBs in the photosphere, UV bursts in the mid/low chromosphere and small (nano-/micro-) flares $\left(10^{6} \mathrm{~K}\right)$ in the upper chromosphere. These results provide new insights into the emergence and build up of the coronal magnetic field and the dynamics and heating of the solar surface and lower atmosphere.
\end{abstract}

Key words: Sun: activity - Sun: atmosphere - Sun: chromosphere - Sun: magnetic fields - Sun: photosphere Sun: UV radiation

Supporting material: animations

\section{Introduction}

The Sun's magnetic field is produced in the solar interior as a result of dynamo action (Parker 1955; Babcock 1963). The dynamo-generated field emerges on the solar surface (photosphere) by magnetic buoyancy and convective motions. Emergence of magnetic flux is a key process leading to the formation of active regions, which host the most intense magnetic activity in the Sun. During flux emergence, magnetic fields rise into the photosphere in the form of magnetic bipoles, which can evolve dynamically to form multi-scale coronal loops over active regions, but, before doing so, they must rid themselves of considerable amounts of mass carried up from below.

Ellerman bombs (EBs, Ellerman 1917) at the photosphere and hot explosions $\left(\sim 8 \times 10^{4} \mathrm{~K}\right)$ in the cool $\left(10^{4} \mathrm{~K}\right)$ solar atmosphere discovered by Interface Region Imaging Spectrograph (IRIS; UV bursts, Peter et al. 2014), are two phenomena typically observed in regions of opposite polarity magnetic fields, most often during flux emergence (Georgoulis et al. 2002; Watanabe et al. 2011; Rutten et al. 2013; Vissers et al. 2013; Reid et al. 2016), but also in the quiet Sun (Rouppe van der Voort et al. 2016). Several studies (Georgoulis et al. 2002; Pariat et al. 2004; Isobe et al. 2007; Matsumoto et al. 2008; Archontis \& Hood 2009; Watanabe et al. 2011; Peter et al. 2014) have supported the idea that reconnection between the opposite polarity fields of interacting bipoles may provide the necessary energy (through conversion from magnetic to kinetic and thermal energy) to accelerate and heat plasma, powering these explosive events and triggering small flares $O$ $\left(10^{6} \mathrm{~K}\right)$ in the solar atmosphere. EBs and UV bursts share many characteristics and both occur in emerging active regions, hence their relation to each other is currently under debate (Vissers et al. 2015; Tian et al. 2016; Danilovic 2017).

In order to produce the particular characteristics of EB emission in the line profile of $\mathrm{H} \alpha$, as well as other lines such as the $\mathrm{Ca}$ II IR and $\mathrm{Ca}$ II $\mathrm{H}$ and $\mathrm{K}$ lines, semi-empirical NLTE models have been constructed. In these, the EB atmosphere is modeled to consist of a "hot spot" of enhanced temperature or density spanning a few hundred kilometers near the temperature minimum (e.g., Berlicki \& Heinzel 2014), or by using a "two cloud" model with an absorbing cool cloud overlying the hot cloud that accounts for the bright $\mathrm{H} \alpha$ wing emission (Hong et al. 2014). These models obtain reasonable fits to observed profiles. Grubecka et al. (2016) extend this type of analysis to the $\mathrm{Mg}$ II $\mathrm{h}$ and $\mathrm{k}$ lines observed with IRIS and conclude that the bright points observed in these lines could be formed in an extended domain spanning the upper photosphere and/or chromosphere (400-750 km).

The studies above show that the temperature increases responsible for line brightening, both for EBs and phenomena at greater heights in the chromosphere such as UV bursts and microflares, presumably are consistent with reconnection processes and associated Joule heating. Idealized, but physically sophisticated 2D magnetohydrodynamic simulations (Xu et al. 2011; Ni et al. 2016), show that reconnection indeed can raise the temperature of the dense plasma to $10^{4} \mathrm{~K}$ or as high as $\gtrsim 8 \times 10^{4} \mathrm{~K}$ depending on plasma- $\beta$ (ratio of the gas pressure to the energy density of the magnetic field) in the low solar atmosphere 100-600 km above the surface.

In the following, we present a realistic 3D numerical model of magnetic flux emergence, which produces the onset of EBs, UV bursts, and small chromospheric flares. We study their nature and address the issue of whether EBs and UV bursts are different events.

In our 3D radiative magnetohydrodynamic (MHD) simulations (Archontis \& Hansteen 2014; Ortiz et al. 2014), a horizontal and uniform magnetic field (flux sheet) emerges from the convection zone to the photosphere, where it forms a network of magnetic bipoles. We find that the interaction of bipolar fields, as driven by resistive emergence into the ambient atmosphere and surface flows, leads to local plasma heating at various heights. Synthetic diagnostics from the numerical model reveal that these heating events match key observed 
characteristics of EBs, UV bursts, and small chromospheric flares.

\section{The Model}

\subsection{Simulations}

The numerical model described in this paper is produced by the Bifrost code (Gudiksen et al. 2011), which solves the magnetohydrodynamic equations on a 3D Cartesian grid. The modeled solar atmosphere spans from $2.5 \mathrm{Mm}$ below the solar surface to $14 \mathrm{Mm}$ above the photosphere, and fills $24 \times 24 \mathrm{Mm}$ in the horizontal direction, i.e., the model covers the upper convection zone to the lower corona. A grid of $504 \times 504 \times 496$ was used with a uniform horizontal resolution of $48 \mathrm{~km}$. The vertical resolution varies from $20 \mathrm{~km} /$ cell in the photosphere, chromosphere, and transition region to nearly $100 \mathrm{~km} /$ cell in the corona and deeper convective layers, where the scale height is much larger. Optically thick radiative transfer and radiative losses from the photosphere and lower chromosphere, including scattering, are implemented using a short characteristics scheme (Hayek et al. 2010). Above the lower chromosphere, radiative losses are parameterized using recipes derived by 1D non-LTE radiative hydrodynamic simulations (Carlsson \& Leenaarts 2012). Field aligned thermal conduction is included with the magnetohydrodynamic equations through operator splitting; the thermal conduction operator is solved using an implicit multi-grid method to allow a reasonably long time-step.

The model is initialized with a weak uniform oblique magnetic field $(<0.1 \mathrm{G})$ that fills the corona, making an inclination angle of $45^{\circ}$ with respect to the $z$-axis. This field is sufficiently weak that it has no effect on the dynamics of the coronal plasma, but its non-vertical direction slows the conductive cooling. The coronal temperature is some $400 \mathrm{kK}$ at the time of flux emergence, overlying a cool chromosphere mainly heated by acoustic shocks (see Ortiz et al. 2014, for further details). At the start of the numerical experiment, a steady state convective equilibrium has been achieved, the chromosphere is in a quasi steady state, while the corona is slowly cooling. Then, a non-twisted horizontal flux sheet of strength $3360 \mathrm{G}$ is injected at the bottom boundary. The magnetic sheet is oriented in the $y$ direction filling the area $[x$; $y]=[3-16 ; 0-24] \mathrm{Mm}$, for a time period of $1^{\mathrm{h}} 45^{\mathrm{m}}$ (this is the same model as that used in Archontis \& Hansteen 2014; Ortiz et al. 2014).

Initially, the magnetic field emerges to the photosphere, where it stops, since the field is no longer carried by convective motions nor is it buoyant. As the field "piles up" from below, the magnetic pressure increases and in certain locations plasma- $\beta$ of the emerging field becomes smaller than one. In these locations magnetic flux elements can emerge through the photosphere and rise into the outer atmosphere above. These rising elements expand and rapidly penetrate and fill the chromosphere and corona, pushing the pre-existing ambient corona aside. The heating events that are described in this paper occur well after (some few thousand seconds) the flux sheet first encounters the photosphere and are a result of self-interactions between the various magnetic elements that have pierced the photosphere and are spreading into the chromosphere and corona.

\subsection{Diagnostics}

The response of the solar atmosphere to the emergence and interactions of the rising magnetic field is studied by computing a number of spectral lines formed in the photosphere and corona.

Synthetic $\mathrm{H} \alpha$ spectra were calculated in 3D non-LTE using the Multi3d code (Leenaarts \& Carlsson 2009). We used a fivelevel plus continuum hydrogen model atom and modeled the Ly $\alpha$ and Ly $\beta$ lines using complete redistribution with a Gaussian profile with Doppler broadening only (Leenaarts et al. 2012a), as an approximation to the more time consuming partial redistribution (PRD) calculations. Radiative transfer calculations were carried out for a total of 24 angles, using the A4 set (Carlson 1963). In addition to the snapshot calculated for display in Figures 1-4, we have calculated $\mathrm{H} \alpha$ emission for a number of snapshots covering a period of $180 \mathrm{~s}$ in order to give an indication of the time evolution line. For these animations (online animated Figures 1 and 2) the calculations were run with reduced horizontal spatial resolution (every second grid point) to reduce the computational burden. For some snapshots, the vertical grid was interpolated to a finer scale to improve convergence times. Both of these procedures were found to have a negligible effect on the line profiles.

The $\mathrm{Mg}$ II synthetic spectra in the vicinity of the $\mathrm{h}$ and $\mathrm{k}$ lines were calculated in $1.5 \mathrm{D}$ non-LTE with PRD using the RH 1.5D code (Uitenbroek 2001; Pereira \& Uitenbroek 2015). In $1.5 \mathrm{D}$, each vertical column from the simulation is treated as an independent 1D atmosphere. This allows for much faster calculations, at the expense of less realistic intensities for wavelengths close to the line cores. This approach is a good approximation for the greater part of the $\mathrm{h}$ and $\mathrm{k}$ line profiles (Leenaarts et al. 2013a), making the computations more tractable than the full 3D PRD calculations (Sukhorukov \& Leenaarts 2016). We used a 10-level plus continuum $\mathrm{Mg}$ II atom (Leenaarts et al. 2013b, same setup as Pereira et al. 2013, although additional lines of other elements were not included) and a hybrid angle-dependent PRD (Leenaarts et al. 2012b).

The Si IV $139.3744 \mathrm{~nm}$ line profiles were also calculated with the RH $1.5 \mathrm{D}$ code using a nine-level model atom including five levels of Si III, three levels of Si IV, and the ground state of Si V. The overlapping Ni II line was included simultaneously using a four-level model atom including the ground term of Ni II as two levels, the upper level of the Ni II $139.3324 \mathrm{~nm}$ line $\left(93 \mathrm{~km} \mathrm{~s}^{-1}\right.$ on the blue side of the Si IV line core) and the ground term of $\mathrm{Ni}$ III. The background continuum of Si I was also included in non-LTE, employing a 16-level model atom with 15 levels of Si I and the ground state of $\mathrm{Si}$ II.

\subsection{Observations}

In order to compare and contrast our synthetic spectra with equivalent observational spectra, we show observations obtained at the Swedish $1 \mathrm{~m}$ Solar Telescope (Scharmer et al. 2003) using the CRISP instrument (Scharmer et al. 2008) on 2015 September 27 at 10:00:39 UT, centered on the emerging active region NOAA 12423. For exploration of the multi-dimensional simulation and observational data cubes, we made much use of CRISPEX (Vissers \& Rouppe van der Voort 2012).

CRISP was running a three-line program at a temporal cadence of $32 \mathrm{~s}$, during which the $\mathrm{H} \alpha$ spectral line was sampled at 15 line positions between $\pm 150 \mathrm{pm}$. The CRISP program 

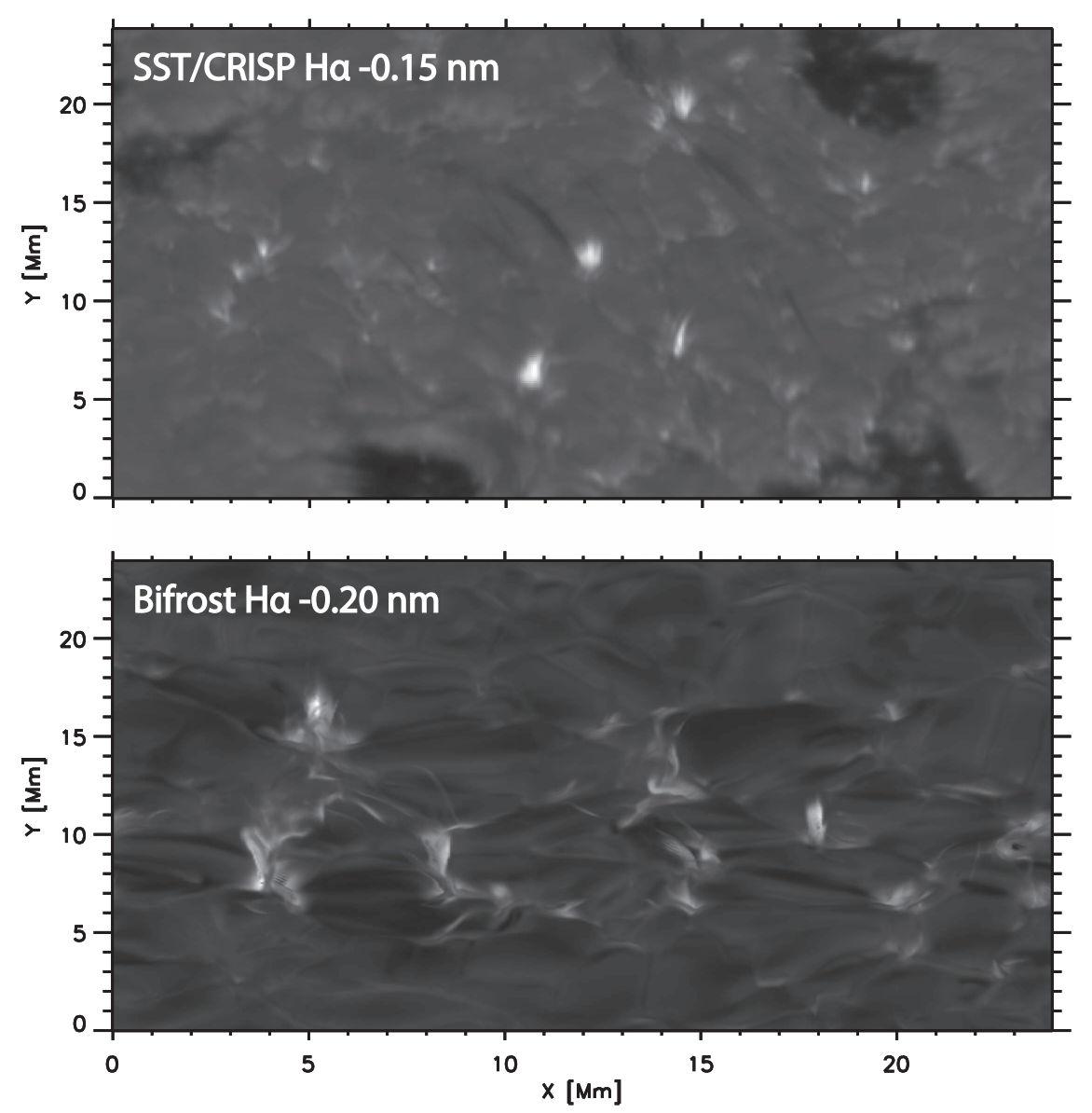

Figure 1. Simulated $\mathrm{H} \alpha$ line wing emission in EBs closely resembles flame-like structures seen in high-resolution ground-based observations. H $\alpha$ intensity in the blue wing (at $-150 \mathrm{pm}$ ) observed with SST/CRISP (top) and at $-200 \mathrm{pm}$ in the synthetic Bifrost images (bottom), at a solar heliocentric angle $\mu=0.5$. The animation of this figure shows the variation of the flame-like structures over a period of some $180 \mathrm{~s}$ (top panel). The bottom panel of the animation shows the synchronous time variation of the synthetic $\mathrm{H} \alpha$ near the line core (at $+50 \mathrm{pm}$ ). Both animations are shown at a heliocentric angle of $\mu=0.5$.

(An animation of this figure is available.)

further includes spectral sampling of the Fe I $617.3 \mathrm{~nm}$ and Ca II $854.2 \mathrm{~nm}$ lines. CRISP data were processed following the CRISPRED data reduction pipeline (de la Cruz Rodríguez et al. 2015), which includes Multi-Object Multi-Frame Blind Deconvolution image restoration (van Noort et al. 2005). The spatial sampling is 0.057 arcsec per pixel, the diffraction limit at $656.3 \mathrm{~nm}$ is 0.14 arcsec. The complete time series has a duration of $02: 43 \mathrm{hr}$, from $07: 47$ to $10: 30 \mathrm{UT}$.

Simultaneous data was obtained with NASA's Interface Region Imaging Spectrograph (IRIS, De Pontieu et al. 2014), which was observing the same active region running an observing program labeled OBS-ID 3620106168 . This program comprises a medium-dense 128-step raster with continuous 0.33 arcsec steps of the 60 arcsec spectrograph slit covering a spatial area of $42.2 \operatorname{arcsec} \times 60$ arcsec at a temporal cadence of 10:53 minutes. The exposure time was $4 \mathrm{~s}$. To increase signal-to-noise, the IRIS spatial sampling was summed on board $(2 \times 2$ pixel binning), so that the original IRIS resolution of 0.166 arcsec spatial sampling was reduced to 0.33 arcsec spatial sampling, while the spectral sampling was reduced from 3 to $6 \mathrm{~km} \mathrm{~s}^{-1}$ sampling. The IRIS data were calibrated to "level 2," i.e., including dark-current, flat-field, and geometric correction (De Pontieu et al. 2014). The slit jaw images (SJI) were corrected for dark-current and flat-field, as well as internal co-alignment drifts.
Alignment between the SST and IRIS data was done through cross-correlation of CRISP Ca II $854.2 \mathrm{~nm}$ wing images and IRIS $279.6 \mathrm{~nm}$ slit jaw images.

\section{Results}

A defining observed characteristic of EBs (Watanabe et al. 2011) is the appearance of rapidly varying extended flames, or jets, jutting out from the photospheric network in the $\mathrm{H} \alpha$ line, most easily seen when looking off disk center (see Figure 1(a)). These synthetic observations can also be compared with observations obtained at the Swedish $1 \mathrm{~m}$ Solar Telescope (Figure 1(b)). It is likely that these flames exist below a "canopy" of dense chromospheric plasma, which prevents any hot emission from the photosphere to be visible in the $\mathrm{H} \alpha$ line core, formed some $2000 \mathrm{~km}$ above the photosphere (Leenaarts et al. 2012a). Thus, EBs appear far from line center on both sides of the $\mathrm{H} \alpha$ line center. Ellerman (1917) reported that the enhanced brightness extends at least $0.4-0.5 \mathrm{~nm}$, and in certain cases attains a width of $3 \mathrm{~nm}$. In the simulations (Figure 1(a)), synthetic images made in the far blue and red wings of $\mathrm{H} \alpha$ show several EBs that are rooted in the photospheric network, located in intergranular lanes between opposite magnetic polarities. They all possess bright strands of dense plasma, which are vertically oriented, and they impart an 


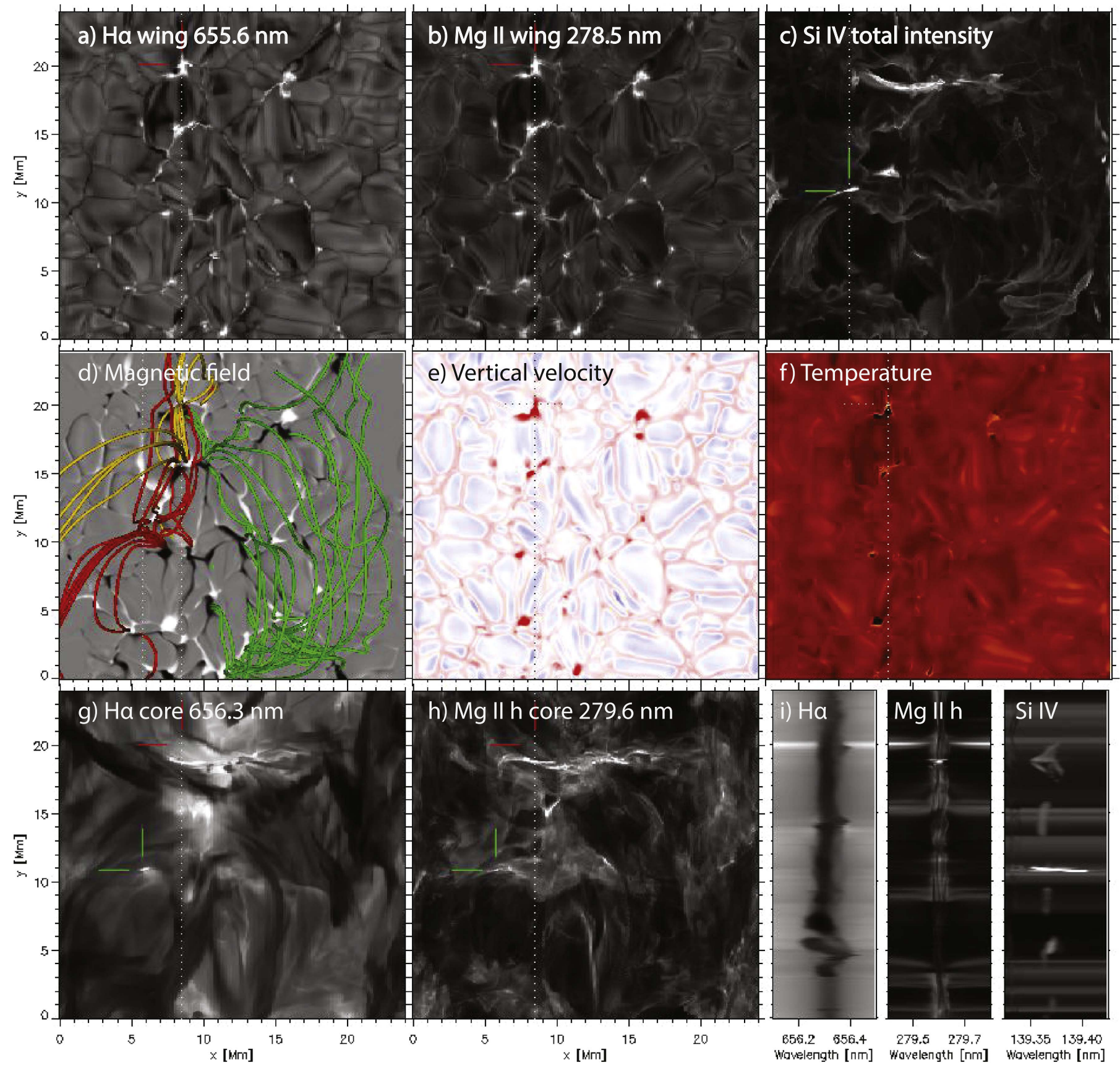

Figure 2. Modeled spectra show signatures that resemble the characteristics of EBs, UV bursts, and small flares. H $\alpha$ line wing (a), Mg II line wing (b), Si IV total intensity (c); photospheric magnetogram $\left(B_{z}\right)$ and selected field lines (d), photospheric velocity $u_{z}(\mathrm{e})$, and temperature (f), $\mathrm{H} \alpha$ line core (g), Mg II line core (h), and line profiles of $\mathrm{H} \alpha, \mathrm{Mg}$ II along $x=8.5$, and $\mathrm{Si}$ IV along $x=6 \mathrm{Mm}$ (i). The red and green cross hairs show the location of a simulated EB and UV bursts, respectively. In panel (d), yellow field lines have been traced from the EB site (indicated by the red orthogonal lines) and red field lines from the vicinity of the small flare. The green field lines have been traced from various magnetic polarities at $z=0 \mathrm{Mm}$ and for $x \geqslant 10 \mathrm{Mm}$. The animation of this figure shows the synthetic H $\alpha$ near the line core (at $+50 \mathrm{pm}$ ) seen from solar heliocentric angle of $\mu=1$ over a period of $200 \mathrm{~s}$ showing formation of opaque dark fibrils over the emerging flux region.

(An animation of this figure is available.)

overall flame-like structure to the EBs. These results come into close agreement with the location, morphology, and dynamics of observed EBs (see also the animation of Figure 1, which shows the time evolution in the line wing and at $+50 \mathrm{pm}$ from the line core).

Synthetic spectroheliograms from the model (Figures 2(a), (b)) show that EBs appear as small (size $1-1.5 \mathrm{Mm}$ ) bright structures in the far wings of $\mathrm{H} \alpha$ and the $\mathrm{Mg}$ II $\mathrm{h}$ and $\mathrm{k}$ lines.
They occur at the interface between opposite polarity fields of adjacent emerging bipoles (Figure 2(d)). Profound downflows (Figure 2(e)) occur on either side of the interface, due to plasma draining along the flanks of the emerging bipoles. The plasma at photospheric heights in the interface is heated to (8-9) $\times 10^{3} \mathrm{~K}$ (Figure 2(f)), giving onset to EBs.

The synthetic EBs are not visible at the line core of the $\mathrm{H} \alpha$ and $\mathrm{Mg}$ II $\mathrm{h}$ lines (Figures $2(\mathrm{~g})$ and $(\mathrm{h})$ ) and they are barely 
$\mathrm{Ha}$
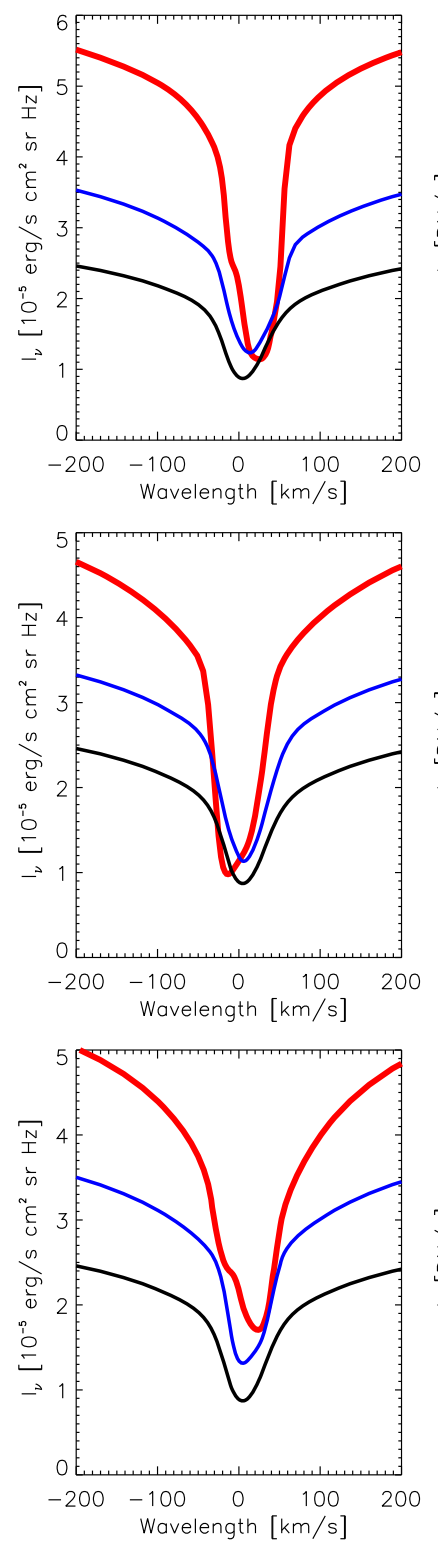

$\mathrm{Mg} \| \mathrm{h}$
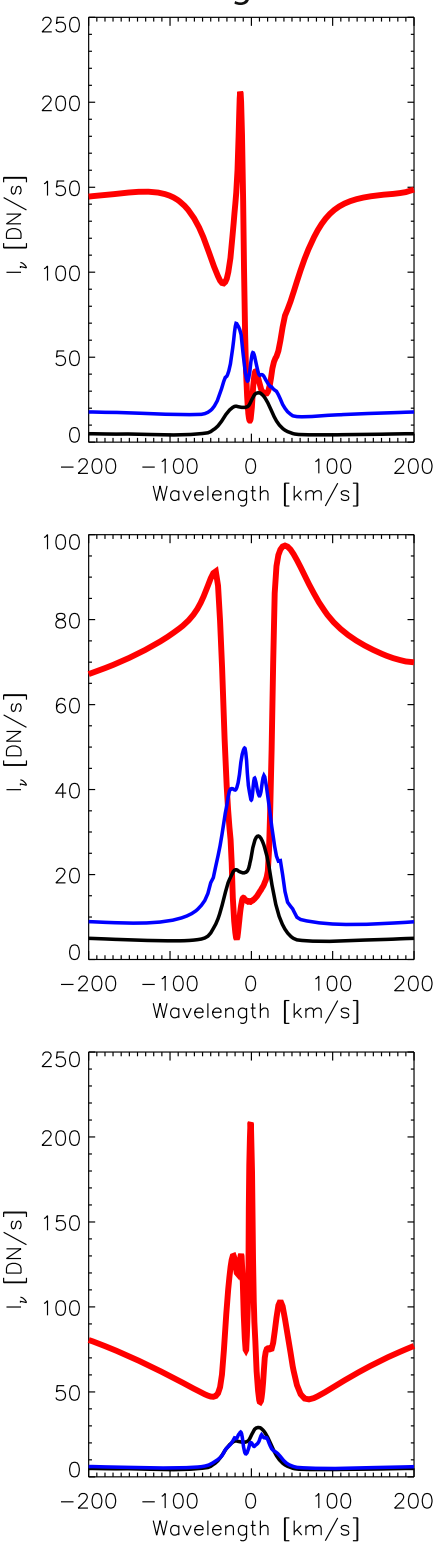

Mg II $279.8 \mathrm{~nm}$
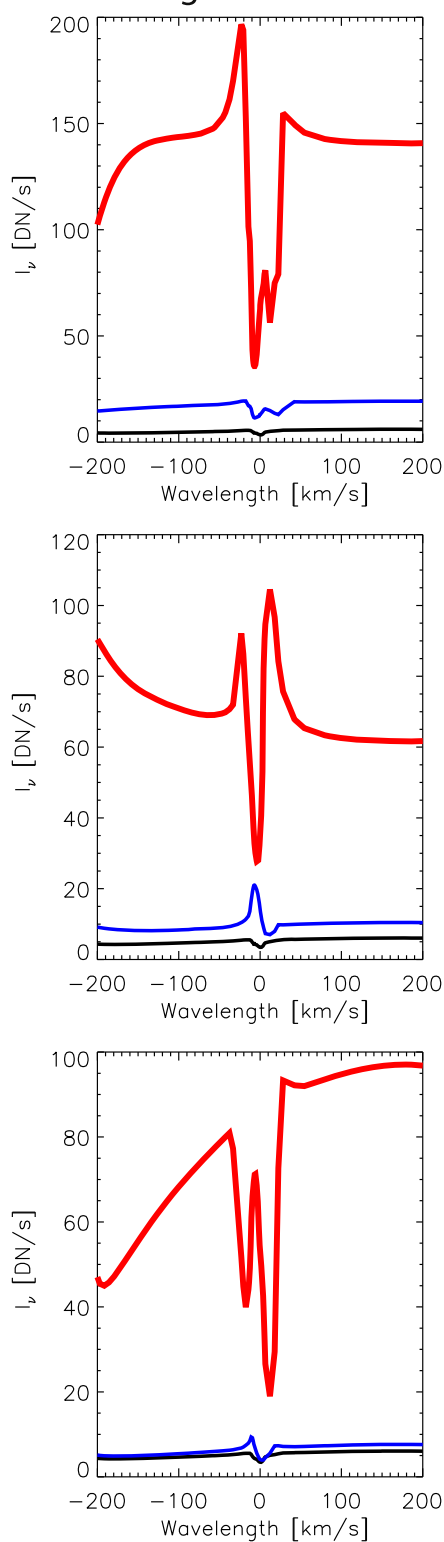

Si IV $139.3 \mathrm{~nm}$

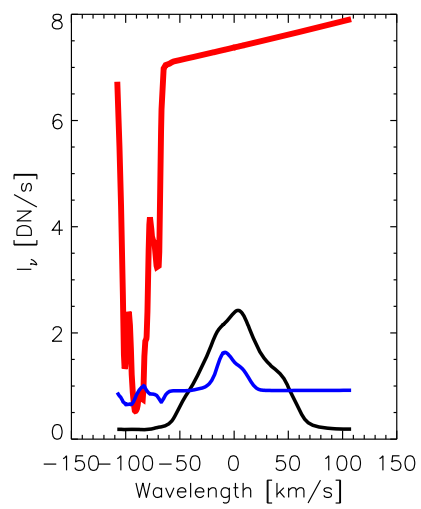

EB1

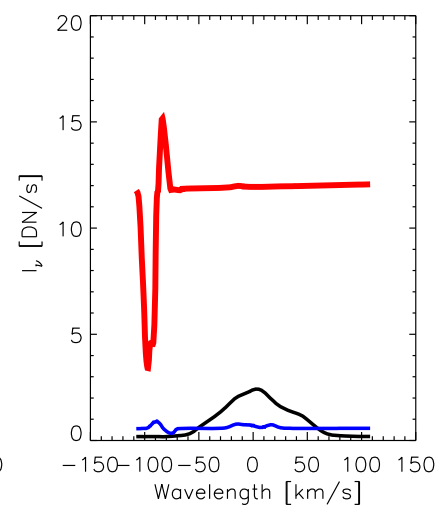

EB2

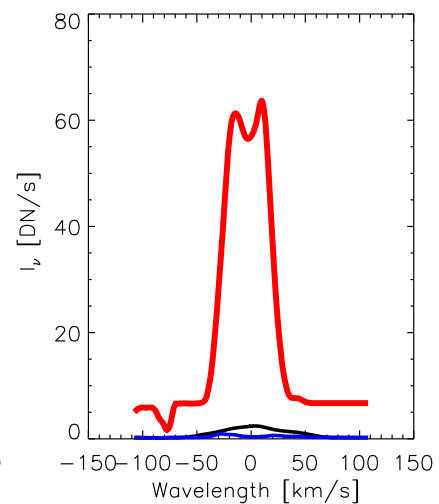

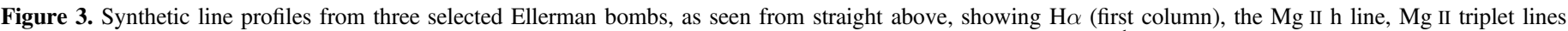

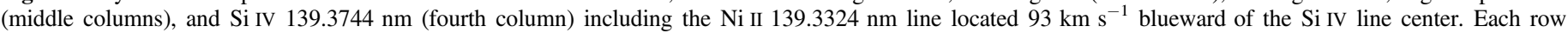

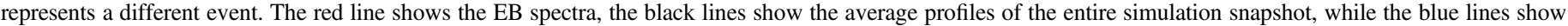
average spectra in the vicinity of the EB.

visible in the Si IV $139.3 \mathrm{~nm}$ band (Figure 2(c)). Just as the $\mathrm{H} \alpha$ core, the line core of the $\mathrm{Mg}$ II $\mathrm{h}$ and $\mathrm{k}$ lines are also mainly formed in the high chromosphere (Leenaarts et al. 2013a). Emission in the Si IV $139.3 \mathrm{~nm}$ line requires temperatures of at least $2 \times 10^{4} \mathrm{~K}$ in the dense photosphere or $(6-8) \times 10^{4} \mathrm{~K}$ in the less dense corona, where the coronal approximation is valid. Thus, they are not responsive to the EBs generated here, which remain below $<10^{4} \mathrm{~K}$ at photospheric heights. In the far wings of $\mathrm{Mg}$ II lines, and at the continuum near the Si IV line, (Figure 2(i)) lessening opacity allows brightening from the EBs to become visible through the overlying canopy, forming a bright "moustache" (see also Figure 3).

On the other hand, the $\mathrm{H} \alpha$ line core (Figure 2(g)) reveals the existence of extended "dark" loops (e.g., at $x=14 \mathrm{Mm}$, $y=0-14 \mathrm{Mm}$, see also the animation of Figure 2, which shows the time evolution in $\mathrm{H} \alpha$ at $+50 \mathrm{pm}$ as seen from above), which are lying along the overarching field lines (e.g., green field lines in Figure 2(d)) that connect distant opposite polarities in the emerging flux region. These newly formed loops carry cool and dense plasma from the photosphere/ chromosphere and they may account for $\mathrm{H} \alpha$ arch-filament systems.

At other locations, there is strong emission at Si IV, with no obvious corresponding signal in $\mathrm{H} \alpha$ (core or wing) and only weak enhancement in Mg II. For instance, the Si IV signal for the brightening at $[x, y]=[8.5,12] \mathrm{Mm}$ (Figures 2(c), (i)) is 200 times stronger than the average, while showing no obvious brightening in $\mathrm{H} \alpha$ nor the $\mathrm{Mg}$ II $\mathrm{h}$ line. The absolute intensity of this brightening is of the same order of magnitude as hot explosions discovered by IRIS (e.g., Peter et al. 2014, see also 
$\mathrm{Ha}$
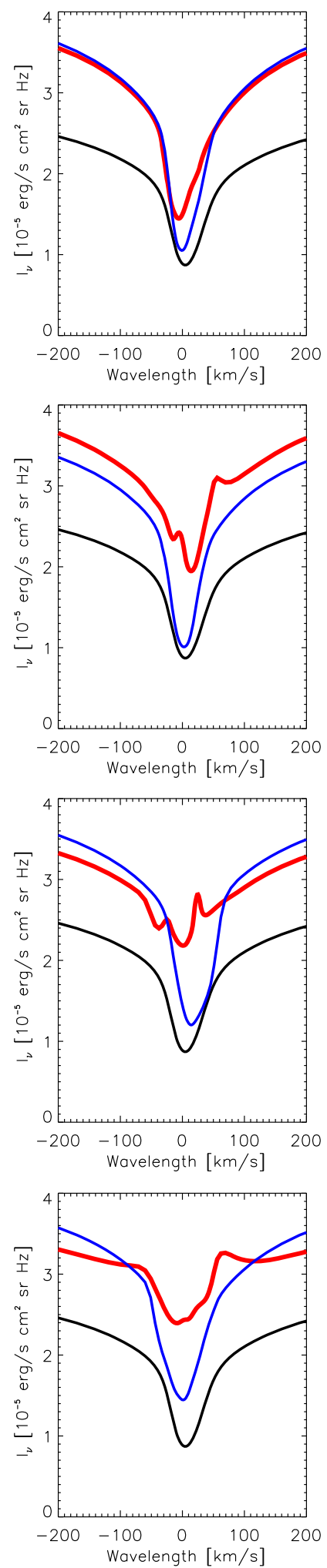

$\mathrm{Mg} \| \mathrm{h}$
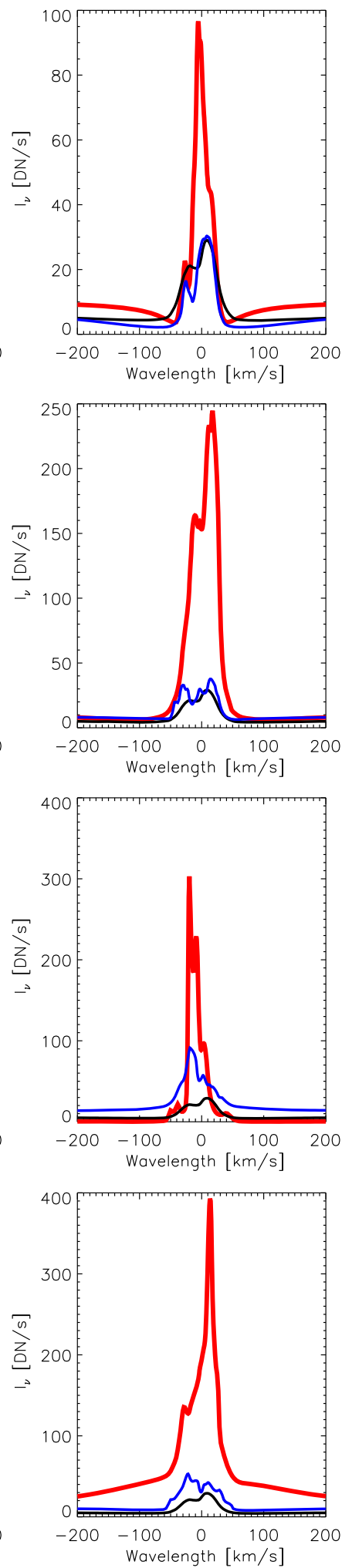

Mg II $279.8 \mathrm{~nm}$
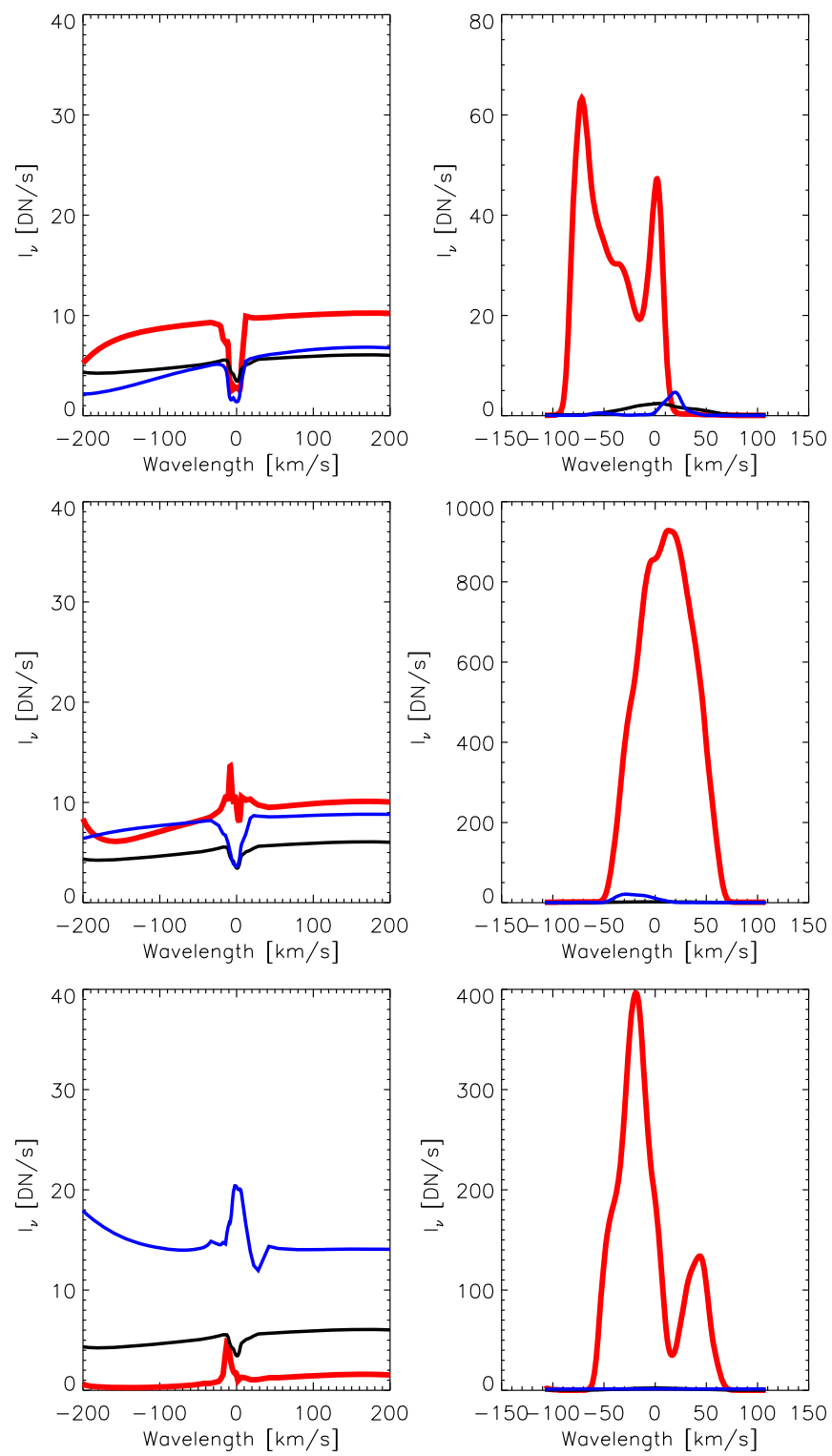

UV3

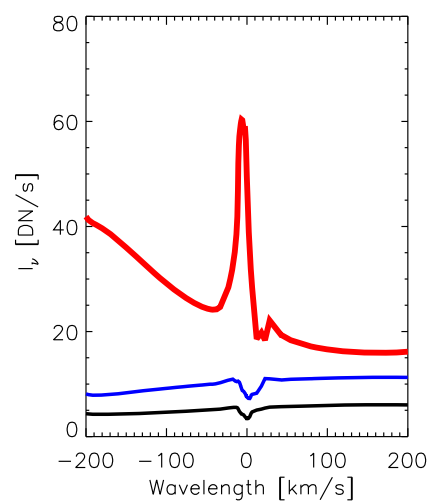

UV2

Si IV $139.3 \mathrm{~nm}$

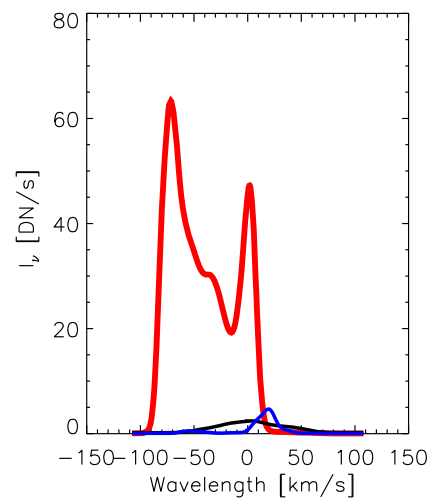

UV1

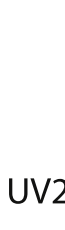

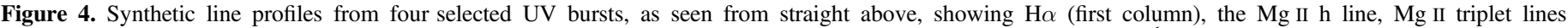

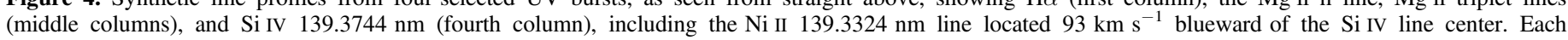

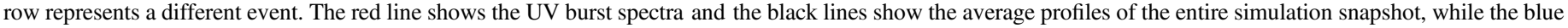
lines show average spectra in the vicinity of the UV burst. 

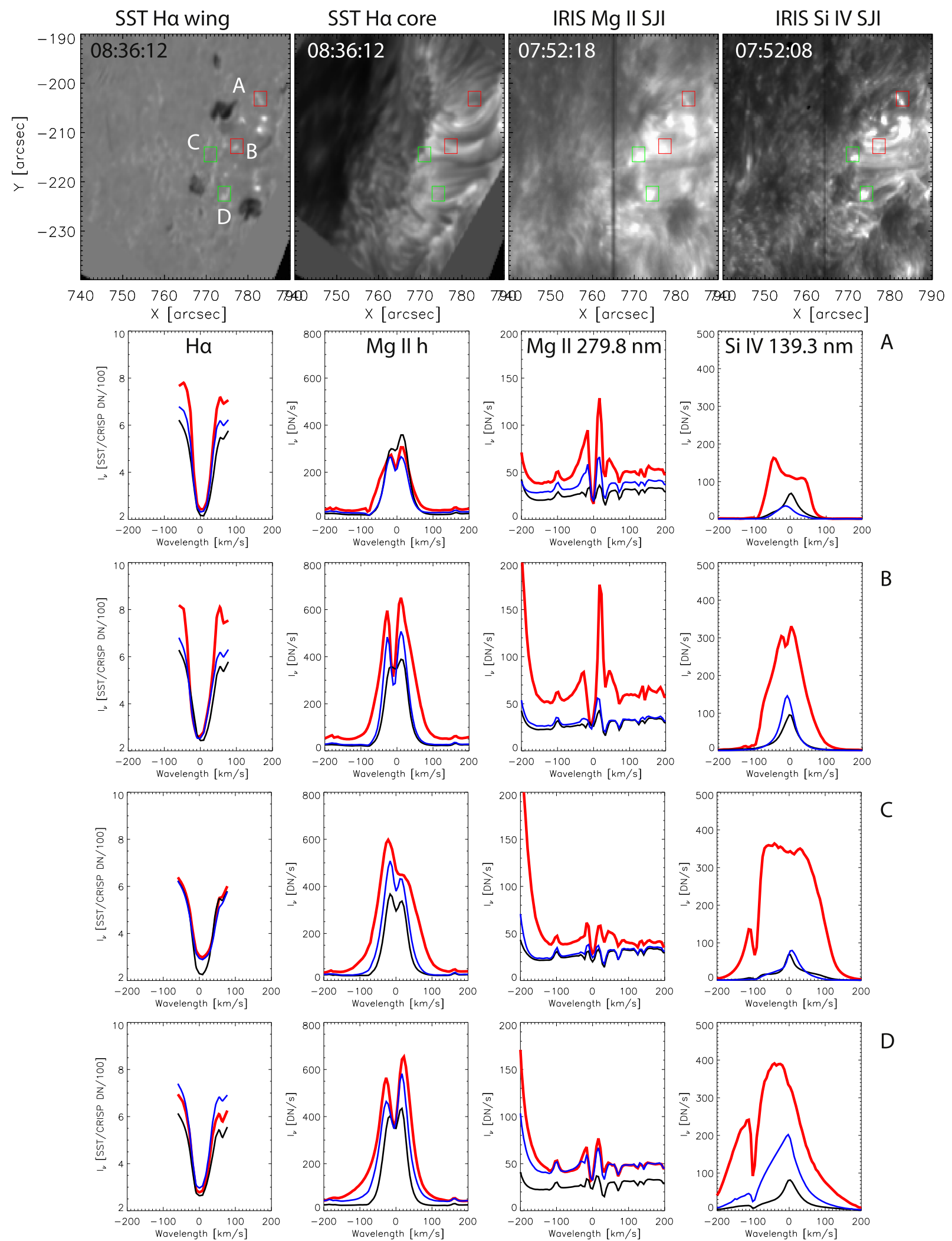

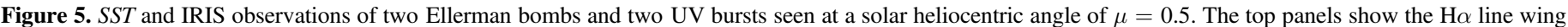

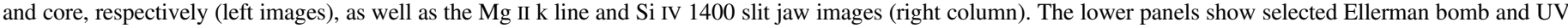

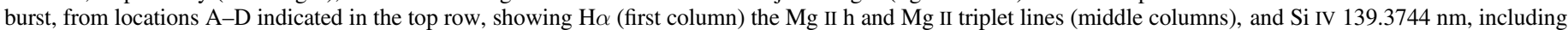

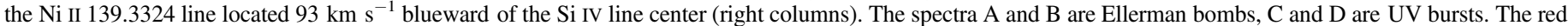

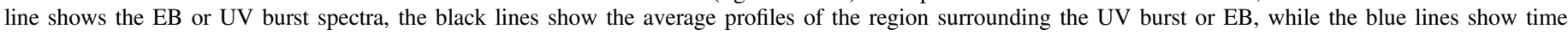
averaged spectra in the vicinity of the EB or UV burst. 

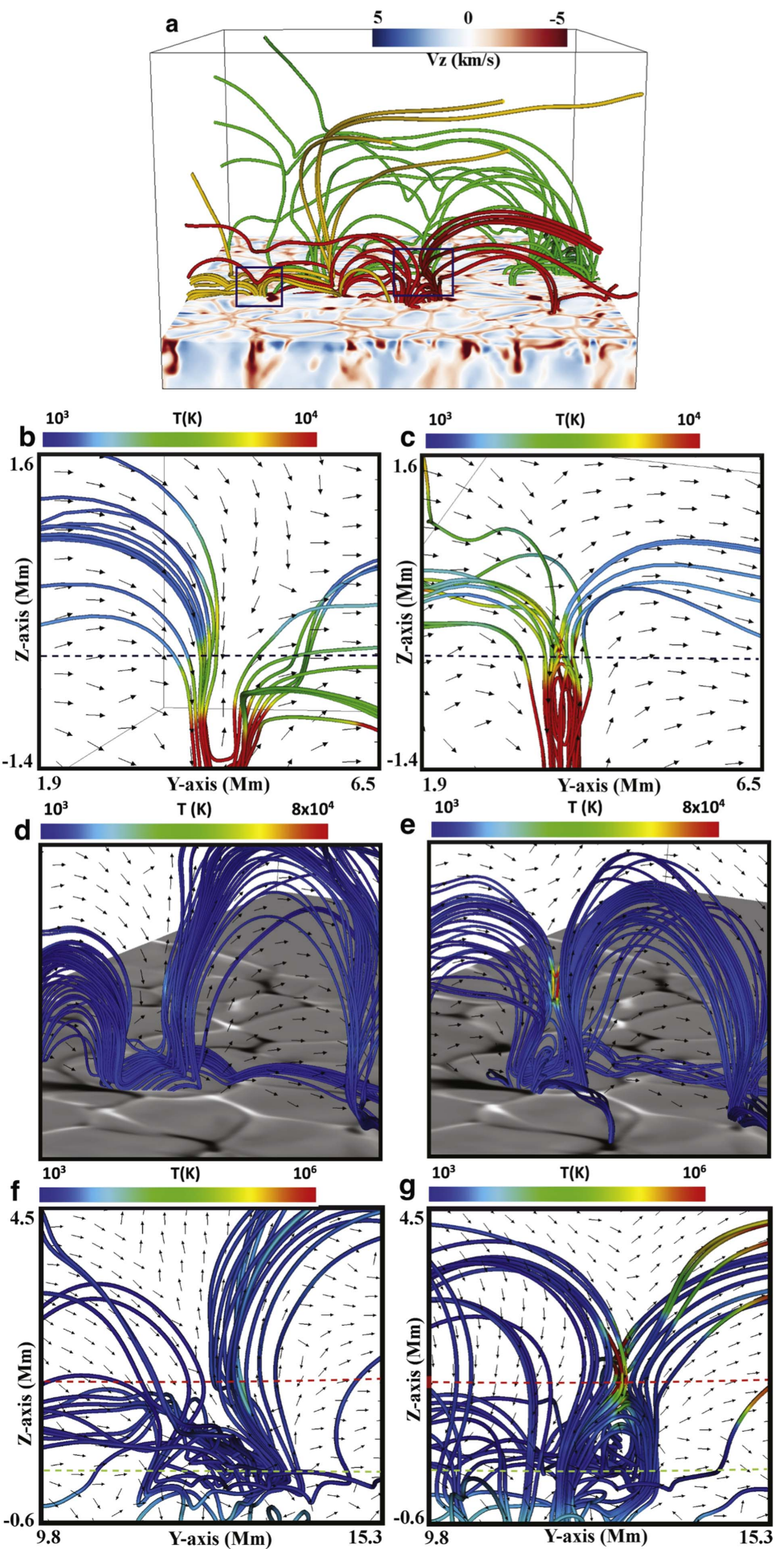

Figure 6. Overall field-line topology and the onset of an EB, UV burst, and a small chromospheric flare. (a) Selected field lines showing the serpentine field at $t=9160 \mathrm{~s}$. Yellow and red field lines have been traced from the areas highlighted by the black insets (left and right, respectively). The image shows the distribution of vertical velocity at the photosphere (red color indicates downflowing material). (b)-(c) Close-up of the field lines, which have been traced from within the left inset in (a), at $t=8800 \mathrm{~s}$ (b) and $t=9160 \mathrm{~s}$ (c). They are colored according to the value of the temperature. The arrows show the projection of the full magnetic field vector at $x=8.4 \mathrm{Mm}$. The horizontal (dashed) line shows the photosphere. The localized temperature enhancement, just above $z=0 \mathrm{Mm}$ and $y \approx 3.5 \mathrm{Mm}$, illustrates the location of the EB. (d)-(e) Emergence of $\Omega$-loops, out of the serpentine field lines ((d), $t=7870 \mathrm{~s})$, leads to expansion and reconnection at the chromosphere ((e), $t=7930 \mathrm{~s})$ and the triggering of a UV burst. The downward-released reconnected field lines build an arcade anchored in the photosphere. The chromospheric plasma at, and close to, the reconnection site (e.g., at the top of the arcade, $y=4.7 \mathrm{Mm}, z=1.3 \mathrm{Mm}$ ) is heated to high temperatures, triggering a UV burst. The horizontal slice shows the $B_{z}$ distribution at the photosphere (white (black) shows positive (negative) $B_{z}$, in the range of $\left[-10^{3}, 10^{3} \mathrm{G}\right]$ ). The arrows show the full magnetic field vector at $x \approx 8 \mathrm{Mm}$. (f)-(g) Close-up of the field lines, which have been traced from within the right inset in (a), at $t=8800 \mathrm{~s}$ (f) and $t=9250 \mathrm{~s}(\mathrm{~g})$. The arrows show the projection of the full magnetic field vector at $x \approx 6 \mathrm{Mm}$. The two horizontal (dashed) lines show heights at $z=1.75 \mathrm{Mm}$ and at $z=0 \mathrm{Mm}$. Nearby emerging loops come into contact and reconnect in a similar manner to the UV burst case. Energy release occurs at the upper chromosphere (around $z=1.8 \mathrm{Mm}, y=13 \mathrm{Mm}$ ), where plasma is less dense, leading to profound plasma heating $\left(10^{6} \mathrm{~K}\right)$ and the onset of a small chromospheric flare. 


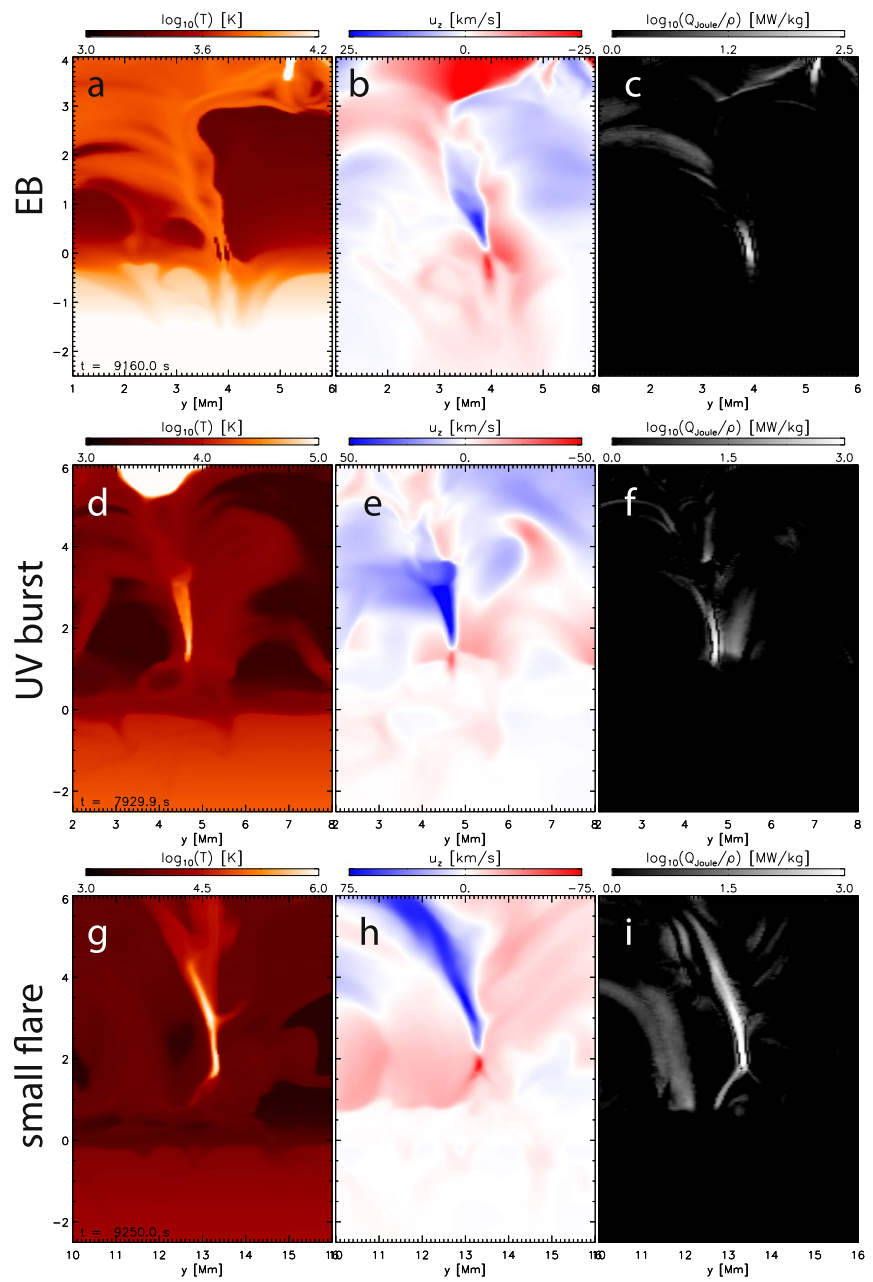

Figure 7. Reconnection in the photosphere and chromosphere heats the local plasma and give rise to fast (supersonic) bi-directional jets at several heights. $2 \mathrm{D}$ cuts at $x=8.43 \mathrm{Mm}, t=9160 \mathrm{~s}(\mathrm{a}-\mathrm{c}), x=8.05 \mathrm{Mm}, t=7930 \mathrm{~s}(\mathrm{~d}-\mathrm{f})$, and $x=6.14 \mathrm{Mm}, t=9250 \mathrm{~s}(\mathrm{~g}-\mathrm{i})$. Shown is temperature (first column), vertical velocity (second column), and Joule dissipation over density (third column). The animation of this figure shows the time development of these variables over a period of $500 \mathrm{~s}$.

(An animation of this figure is available.)

Figure 5). The Si IV line profile is also very broad in this location, as discussed below, and thus, these events may correspond to the observed UV bursts.

Moreover, there are brightenings with very strong emission at $\mathrm{Si}$ IV and considerable emission at the line core of $\mathrm{H} \alpha$ and $\mathrm{Mg}$ II $\mathrm{h}$ line (e.g., at the location indicated by the green orthogonal lines, Figures 2(c), (g), and (h)). This is where plasma at the upper chromosphere is heated to high temperatures $O\left(10^{6}\right) \mathrm{K}$. These explosions are associated with the onset of small flares (Figures 6 and 7).

\subsection{Detailed Spectra}

The observational signatures of EBs and UV bursts are quite complicated. To give further insight, we present several synthetic line spectra from reconnection events occurring in the photosphere and in the middle to upper chromosphere and compare them with observed spectra taken at the SST and with IRIS.

In addition to the "flame-like" structure seen in the wings of $\mathrm{H} \alpha$, a number of "secondary" EB characteristics have been reported (Vissers et al. 2015). In Figure 3, we present the spectra of several simulated EBs in $\mathrm{H} \alpha$, the $\mathrm{Mg}$ II h line near $279.6 \mathrm{~nm}$, the $\mathrm{Mg}$ II triplet lines near $279.8 \mathrm{~nm}$, and the Si IV $139.3744 \mathrm{~nm}$ line (including the Ni II blend at $139.3324 \mathrm{~nm}$ ). In Figure 4, the spectra of synthetic UV bursts are shown.

Figure 3 shows that the $\mathrm{H} \alpha$ line core is unaffected by the presence of the EB, mainly due to the existence of an overlying chromospheric canopy above the EB. For all cases, we find that the line wings are significantly enhanced; by roughly a factor of two when seen from directly above, and that this enhancement extends sme $0.1 \mathrm{~nm}$ or more on either side of the line core. As for $\mathrm{H} \alpha$, the $\mathrm{Mg}$ II line core is opaque and emission in the core largely stems from the overlying (usually cooler) chromosphere, but the overlying material becomes transparent as one moves away from the central core and we see brightenings, sometimes asymmetric, in the outer core and wings of the $\mathrm{Mg}$ II $\mathrm{h}$ line, as well as large brightening in the continuum surrounding the line.

In most of the simulated EBs, we also find asymmetric emission in the Mg II triplet lines near $279.8 \mathrm{~nm}$. Emission in these lines is observationally rare and indicates that the chromospheric temperature is high at low chromospheric heights (Pereira et al. 2015).

In general, we do not find any significant enhancement in the Si IV $139.3744 \mathrm{~nm}$ line above our simulated EBs. In the cases that we have studied, we usually find enhanced continuum emission and absorption in the Ni II line (which sometimes shows a complicated line structure indicative of large relative flows in the chromosphere). In some instances, we find enhanced Si IV emission in the same locations as the EB, but these are often dominated by heating events, unrelated to the $\mathrm{EB}$, at greater heights in the chromosphere. Though the emission is unrelated, the magnetic field structure of these brightenings is connected, forming active sites in the larger magnetic flux structure that is emerging through the photosphere. This possible connection between disparate emitting sites has also been noted observationally (Qiu et al. 2000). Note that when seen from the side, we occasionally see that the upper part of the EB does produce emission in Si IV, but in every instance we have found that this is dominated by emission from greater heights when seen from above.

Simulated line profiles from reconnection events that occur higher in the atmosphere are shown in Figure 4. The $\mathrm{H} \alpha$ core emission from these events depends on event height and strength, while the wing emission is always unresponsive. The line core can either be unresponsive, or in some cases show significant brightening and complex structure for the strongest most violent events-perhaps forming a counterpart to small flares.

The Mg II $\mathrm{h}$ line core emission is enhanced by a large factor and the core line profile shows quite complicated structure reflecting the high velocity jets that form the core of these reconnection events. As for $\mathrm{H} \alpha$, the $\mathrm{Mg}$ II triplet line emission does not always respond to these UV bursts, but occasionally shows asymmetric emission profiles and emission.

On the other hand, the Si IV emission is greatly enhanced in some cases. It can increase as much as three orders of magnitude over the average value and it reflects intensities comparable to those measured with IRIS. The line profile of the Si IV lines is quite complex, but, in general, shows bidirectional Doppler shifts with amplitudes of up to $100 \mathrm{~km} \mathrm{~s}^{-1}$. The Si IV line profile is very broad, of the order 
of $100 \mathrm{~km} \mathrm{~s}^{-1}$, indicating highly supersonic velocities for the lines formed at the greatest heights (the speed of sound is of the order of $30 \mathrm{~km} \mathrm{~s}^{-1}$ at the temperature of Si IV emission). The cases of high Si IV intensities we report here are all formed in the middle to upper chromosphere and we find that the line has moderate opacity, with $\tau \sim 10$. We would expect Si IV to be even thicker, were the line formed deeper in the atmosphere, e.g., in connection with an EB.

To make a comparison between the simulated spectra and the observations, we present line profiles of a number of EBs and UV bursts observed with the SST and IRIS on 2015 September 27 starting at 07:52:39 UT in the vicinity of active region NOAA 12423 . Two sets of two spectra are presented in Figure 5.

In the first two cases, (A) and (B), two EBs were selected by inspecting the $\mathrm{H} \alpha$ wing spectroheliogram for bright flame-like structures and then plotting the spectra of $\mathrm{H} \alpha$ and the concurrent IRIS UV lines in the same region. In addition, to give an idea of the temporal variability of these lines in that region, we plot the average profile and spectra at a temporal cadence of some 22 minutes for the entire observational run lasting some $4.5 \mathrm{hr}$. $\mathrm{H} \alpha$ responds as expected, with bright wings and unresponsive core emission. The $\mathrm{Mg}$ II $\mathrm{h}$ line core emission is unresponsive, while the h2 peaks are broadened, sometimes (case B) showing increased emission, as is also the case in the continuum where the intensity increases by almost a factor of two. The Mg II triplet lines show both enhanced emission and asymmetric profiles. In the Si IV line, we find broad $O\left(200 \mathrm{~km} \mathrm{~s}^{-1}\right)$ profiles and enhanced emission, but less than in the UV burst cases discussed below.

Likewise, cases (C) and (D) were selected by searching for bright Si IV SJI emission and then plotting $\mathrm{H} \alpha$ and the other IRIS lines concurrently, including average and time variability spectra for reference. The Si IV line spectra are extremely broad $O\left(300 \mathrm{~km} \mathrm{~s}^{-1}\right)$, sometimes asymmetric (case D), and $\mathrm{Ni} \mathrm{II}$ absorption is strong in both examples. $\mathrm{H} \alpha$ emission, on the other hand, seems quite unresponsive. We note that there is an EB-like brightening that occurs in case D, but it appears some 66 minutes after the original Si IV brightening and is slightly offset from the Si IV SJ emission, thus likely not representing the same event. $\mathrm{Mg}$ II $\mathrm{h}$ line emission is enhanced, both in the $\mathrm{h} 2$ peaks and (case $\mathrm{C}$ ) in the $\mathrm{h} 3$ line core, as well as being asymmetric. The continuum emission is slightly enhanced. The $\mathrm{Mg}$ II triplet lines are in emission, though to a much smaller extent than as seen in the EB cases (A and B).

The time cadence of this observation is, unfortunately, too low to show the time evolution of these events clearly, obtaining such data should be a goal for future studies.

\subsection{Dynamics and Energetics}

To understand the mechanism for the onset of the EBs, UV bursts, and small flares in our experiment, we study the topology of the magnetic field (Figure 6(a)). Initially, the subphotospheric magnetic field is pulled up by convective upflows and buoyancy (where the field is strong) and it is dragged down by downdrafts, developing an undulating shape. When the strongest field breaks through the surface, small (1-2 Mm) magnetic bipoles appear at the photosphere. Eventually, the field expands into the ambient atmosphere forming $\Omega$-like loops, which may reconnect with each other, creating longer magnetic structures (i.e., the arch-filaments shown in Figure 2). As a result, the emerging magnetic field develops an overall "sea-serpent" configuration of loops with increasingly larger scales with height.

The EBs are formed at the photosphere, between the opposite polarity field lines of adjacent emerging loops (Figures 6(b), (c)). As the U-like parts of the undulating field are pulled down by convective downflows, the nearby legs of the adjacent loops are pressed together and their field lines reconnect, which leads to local plasma heating and the onset of EBs. A side effect of this process is the unloading of mass from the serpentine field (Isobe et al. 2007; Archontis \& Hood 2009; Tortosa-Andreu \& Moreno-Insertis 2009; Cheung et al. 2010). Reconnection above the density-loaded U-dips, forms twisted magnetic structures (O-shaped, as projected onto the $y z$-plane), which are dragged into the convection zone, together with their attached heavy plasma. Some amount of cool plasma is carried upward with the expanding field forming the opaque chromospheric canopy.

A similar mechanism occurs at larger heights, powering UV bursts (at $z \sim 1.5 \mathrm{Mm}$, Figures 6(d), (e)) and small flares (at $z \sim 1.8 \mathrm{Mm}$, Figures $6(\mathrm{f}),(\mathrm{g}))$. The lateral expansion of adjacent $\Omega$-loops, brings their oppositely directed stressed fields into contact, leading to reconnection at their interface and heating of chromospheric plasma to high temperatures $\left((7-8) \times 10^{4} \mathrm{~K}\right.$ for the UV bursts and $\sim 1 \mathrm{MK}$ for the small flares).

Figure 7 shows vertical cuts at the locations of an EB $(a-c)$, $\mathrm{UV}$ burst $(\mathrm{d}-\mathrm{f})$ and small chromospheric flare $(\mathrm{g}-\mathrm{i})$. In all cases, strong currents are built up at the interfaces between the interacting magnetic fields. Reconnection at the current layers leads to plasma heating via Joule dissipation (panels c, $\mathrm{f}, \mathrm{i}$, and the online animated version, which shows the time evolution of these quantities) and to the emission of bi-directional flows (b, e, h) with velocities comparable to the local Alfvén speed. Note that the velocities are asymmetric, with the upward propagating portion having substantially higher velocity (see, e.g., Libbrecht et al. 2016). The aforementioned events have lifetimes of at least $120-180 \mathrm{~s}$.

Above the EB, dense $\left(\sim 10^{-8} \mathrm{~g} \mathrm{~cm}^{-3}\right)$ and cool $\left(\sim 7.5 \times 10^{3} \mathrm{~K}\right)$ plasma is emitted along the reconnection jet with a velocity of $\sim 20 \mathrm{~km} \mathrm{~s}^{-1}$. Some $1300 \mathrm{~km}$ higher, at the site of the UV burst, dense chromospheric material $\left(\sim 5 \times 10^{-13} \mathrm{~g} \mathrm{~cm}^{-3}\right)$ is heated to $\sim 7.5 \times 10^{4} \mathrm{~K}$ (d). Before the burst, the local (unperturbed) plasma has similar density but it is 10 times cooler. The total intensity computed in the Si IV line also shows that the UV burst occurs at chromospheric heights $(z=1-2 \mathrm{Mm})$ in this case. The reconnection jets run with speeds of $40-70 \mathrm{~km} \mathrm{~s}^{-1}$ (e). The upward jet shoots cool $\left((1-2) \times 10^{4} \mathrm{~K}\right)$ chromospheric plasma to coronal heights (reaches $z \geqslant 4 \mathrm{Mm}$ at $7980 \mathrm{~s}$ ). The downward jet terminates at the apex of the post-reconnection arcade (at $z \sim 1 \mathrm{Mm}$ ), where the plasma is heated by compression. Our results suggest that most UV bursts may originate from low/midchromospheric plasma and are not necessarily photospheric as previously suggested (Peter et al. 2014).

When stronger magnetic fields $(50-100 \mathrm{G})$ reconnect in the upper chromosphere (e.g., $z \sim 2.1 \mathrm{Mm}$, panel g), the local plasma $\left(\sim(1-1.5) \times 10^{-13} \mathrm{~g} \mathrm{~cm}^{-3}\right)$ is heated to even higher temperatures $\left(O\left(10^{6}\right) \mathrm{K}\right)$. The local plasma- $\beta$, within the volume $\left(\sim 700 \times 500 \times 1000 \mathrm{~km}^{3}\right)$ of the profound heating, is $\sim 0.02$, which explains the increase of temperature by a factor of $\sim 1 / \beta$. The hot and dense reconnection jets are emitted upward (up to $z \sim 4 \mathrm{Mm}$ ) and downward (to $\sim 1.5 \mathrm{Mm}$ ) with maximum speeds of some $80 \mathrm{~km} \mathrm{~s}^{-1}$. 
The energetics of our synthetic EBs, UV bursts, and small flares can be estimated as follows. The (average) magnetic field close to the EB described in the main text is $\sim 600 \mathrm{G}$. The total magnetic energy stored in the EB's volume $(\sim 500 \mathrm{~km} \times 500 \mathrm{~km} \times 400 \mathrm{~km})$ is $\sim 1.5 \times 10^{20} \mathrm{~J}$. The total thermal energy content is $\sim 10^{20} \mathrm{~J}$. These values lie within the expected energy range for EBs. The magnetic field strength near the UV burst is 15-20 G and plasma $\beta \approx 0.1$. Thus, the total magnetic energy dumped in the burst's volume $(\sim 700 \mathrm{~km} \times 500 \mathrm{~km} \times 800 \mathrm{~km})$ and the involved thermal energy are up to $3.8 \times 10^{17} \mathrm{~J}$ and $(2-3) \times 10^{17} \mathrm{~J}$ respectively, which are much less than those computed for the synthetic EB.

In the upper chromosphere, the flaring event described in the main text has a magnetic field of 50-100 G. The total stored energy is $O\left(10^{18}\right) \mathrm{J}$, which lies within the nanoflare energy regime. According to the above values, the heated plasma around the reconnection site may account for an EUV flare and the emitted jet for an EUV jet.

\section{Discussion and Conclusions}

The simulations and synthetic diagnostics presented here show that many, though perhaps not all, observed characteristics of EBs and UV bursts can be reproduced within the confines of a relatively simple scenario, where an untwisted flux sheet is allowed to emerge through the solar atmosphere and expand into the overlying atmosphere.

While $\mathrm{H} \alpha$ and the $\mathrm{Mg}$ II triplet lines appear very similar to the observed spectra presented in Figure 5, the $\mathrm{Mg} \mathrm{II} \mathrm{h}$ (and k) lines are not as easy to characterize. The observed EB spectra show enhanced h2 and slightly enhanced h3 peaks and line wings. The synthetic spectra show strongly enhanced wings but only sometimes enhanced cores and strong asymmetries. We suspect that the difference in viewing angle $(\mu=1$ for the simulations, $\mu=0.5$ for the observations) is the cause of the difference in the wings: Grubecka et al. (2016) observe EBs close to disk center and they observe strongly enhanced wings. The observed UV burst spectra show strong enhancement for the $\mathrm{h} 2$ cores, sometimes enhanced $\mathrm{h} 3$ and intensity increases in the continuum. This is not too different from what is found in the synthetic spectra, though the increase in core width is much greater in many observed spectra. It should be noted that the variations in $\mathrm{Mg}$ II $\mathrm{h}$ and $\mathrm{k}$ line response to UV bursts is very large, as can be ascertained also from other sources (Vissers et al. 2015; Grubecka et al. 2016).

The synthetic Si IV spectra show an intriguing similarity to the observed line profiles, but there are also differences that could point the way toward a better understanding of the solar atmosphere in general and EBs and UV bursts in particular. We found no Ni II absorption in those Si IV line profiles that extend to more than $100 \mathrm{~km} \mathrm{~s}^{-1}$ in the blue. This could be due to several factors: (1) there is not enough cool material above the sites of strong $\mathrm{Si}$ IV emission in the model to give sufficient opacity in the Ni II line, (2) the simulated EBs and deep UV bursts do not heat the denser low atmosphere material to high enough temperatures to cause Si IV emission, and/or (3) the velocities in deeper UV bursts are not great enough to give significant emission at a blueshift of $100 \mathrm{~km} \mathrm{~s}^{-1}$ in locations where the opacity of the overlying material is great enough. We note that reconnection in this model is mediated by the use of an artificial hyper-diffusive operator, necessary to prevent the collapse of current sheets to smaller widths than the grid size $(48 \mathrm{~km})$. The effective diffusivity on the real Sun may allow current sheets to become thinner, with attendant higher temperatures. Not covered in this study is the possibility that reconnection, even at low heights in the atmosphere, produces non-thermal electrons in sufficient quantities to perturb emission. Ding et al. (1998) and Fang et al. (2006) show that this process may be of interest. In particular, these authors find that the temperature rise needed to explain EB emission is somewhat lower than that found in models without particle beams. On the other hand, it is also likely that since EBs have a fairly large vertical extent, also with respect to Joule heating (see Figure 7), a slightly altered magnetic topology could lead to Si IV emission from the upper part of EBs rooted in the photosphere, even in models with relatively poor resolution, such as this one. The study of EBs and UV bursts, by comparing observational and synthetic diagnostics, thus presents a unique opportunity to study reconnection, and the physical parameters controlling reconnection, at many heights in the solar atmosphere.

In order to form the coronal magnetic field, emerging magnetic flux must break through the photosphere, while ridding itself of considerable mass. This process needs reconnection in order to proceed, occurring at several heights as the field rises into the upper atmosphere forming continually longer loops. The above results reveal that reconnection between stressed magnetic fields in an emerging flux region can trigger three types of solar explosive events (EBs, UV bursts, and small chromospheric flares) of different origins. We conjecture that a similar mechanism can form a continuum of explosions across the solar atmosphere, depending on the local properties of the plasma and the amount of available energy involved in the process whose visibility is determined by the amount of overlying opaque (and cool) material.

This research was supported by the Research Council of Norway and by the European Research Council under the European Union's Seventh Framework Programme (FP7/ 2007-2013)/ERC Grant agreement no. 291058. The simulations have been run on clusters from the Notur project, and the Pleiades cluster through the computing project s1061 from NASA's High End Computing Capability (HECC). We thankfully acknowledge the computer and supercomputer resources of the Research Council of Norway through grant $170935 /$ V30 and through grants of computing time from the Programme for Supercomputing, The Swedish $1 \mathrm{~m}$ Solar Telescope is operated on the island of La Palma by the Institute for Solar Physics of Stockholm University in the Spanish Observatorio del Roque de los Muchachos of the Instituto de Astrofísica de Canarias. IRIS is a NASA small explorer mission developed and operated by LMSAL with mission operations executed at NASA Ames Research center and major contributions to downlink communications funded by ESA and the Norwegian Space Centre.

\section{References}

Archontis, V., \& Hansteen, V. 2014, ApJL, 788, L2

Archontis, V., \& Hood, A. W. 2009, A\&A, 508, 1469

Babcock, H. W. 1963, ARA\&A, 1, 41

Berlicki, A., \& Heinzel, P. 2014, A\&A, 567, A110

Carlson, B. G. 1963, in Methods in Computational Physics, Vol. 1 ed. B. Alder, S. Fernbach, \& M. Rotenberg (New York: Academic)

Carlsson, M., \& Leenaarts, J. 2012, A\&A, 539, A39

Cheung, M. C. M., Rempel, M., Title, A. M., \& Schüssler, M. 2010, ApJ, 720,233

Danilovic, S. 2017, arXiv:1701.02112 
de la Cruz Rodríguez, J., Löfdahl, M. G., Sütterlin, P., Hillberg, T., \& Rouppe van der Voort, L. 2015, A\&A, 573, A40

De Pontieu, B., Title, A. M., Lemen, J. R., et al. 2014, SoPh, 289, 2733

Ding, M. D., Henoux, J.-C., \& Fang, C. 1998, A\&A, 332, 761

Ellerman, F. 1917, ApJ, 46, 298

Fang, C., Tang, Y. H., Xu, Z., Ding, M. D., \& Chen, P. F. 2006, ApJ, 643,1325

Georgoulis, M. K., Rust, D. M., Bernasconi, P. N., \& Schmieder, B. 2002, ApJ, 575,506

Grubecka, M., Schmieder, B., Berlicki, A., et al. 2016, A\&A, 593, A32

Gudiksen, B. V., Carlsson, M., Hansteen, V. H., et al. 2011, A\&A, 531, A154

Hayek, W., Asplund, M., Carlsson, M., et al. 2010, A\&A, 517, A49

Hong, J., Ding, M. D., Li, Y., Fang, C., \& Cao, W. 2014, ApJ, 792, 13

Isobe, H., Tripathi, D., \& Archontis, V. 2007, ApJL, 657, L53

Leenaarts, J., \& Carlsson, M. 2009, in ASP Conf. Ser. 415, The Second Hinode Science Meeting: Beyond Discovery-Toward Understanding, ed. B. Lites et al. (San Francisco, CA: ASP), 87

Leenaarts, J., Carlsson, M., \& Rouppe van der Voort, L. 2012a, ApJ, 749, 136

Leenaarts, J., Pereira, T., \& Uitenbroek, H. 2012b, A\&A, 543, A109

Leenaarts, J., Pereira, T. M. D., Carlsson, M., Uitenbroek, H., \& De Pontieu, B. 2013a, ApJ, 772, 89

Leenaarts, J., Pereira, T. M. D., Carlsson, M., Uitenbroek, H., \& De Pontieu, B. 2013b, ApJ, 772, 90

Libbrecht, T., Joshi, J., de la Cruz Rodríguez, J., Leenaarts, J., \& Asensio Ramos, A. 2016, arXiv:1610.01321

Matsumoto, T., Kitai, R., Shibata, K., et al. 2008, PASJ, 60, 577

Ni, L., Lin, J., Roussev, I. I., \& Schmieder, B. 2016, ApJ, 832, 195

Ortiz, A., Bellot Rubio, L. R., Hansteen, V. H., de la Cruz Rodríguez, J., \& Rouppe van der Voort, L. 2014, ApJ, 781, 126

Pariat, E., Aulanier, G., Schmieder, B., et al. 2004, ApJ, 614, 1099
Parker, E. N. 1955, ApJ, 122, 293

Pereira, T. M. D., Carlsson, M., De Pontieu, B., \& Hansteen, V. 2015, ApJ, 806, 14

Pereira, T. M. D., Leenaarts, J., De Pontieu, B., Carlsson, M., \& Uitenbroek, H. 2013, ApJ, 778, 143

Pereira, T. M. D., \& Uitenbroek, H. 2015, A\&A, 574, A3

Peter, H., Tian, H., Curdt, W., et al. 2014, Sci, 346, 1255726

Qiu, J., Ding, M. D., Wang, H., Denker, C., \& Goode, P. R. 2000, ApJL, 544, L157

Reid, A., Mathioudakis, M., Doyle, J. G., et al. 2016, ApJ, 823, 110

Rouppe van der Voort, L. H. M., Rutten, R. J., \& Vissers, G. J. M. 2016, A\&A, 592, A100

Rutten, R. J., Vissers, G. J. M., Rouppe van der Voort, L. H. M., Sütterlin, P., \& Vitas, N. 2013, JPhCS, 440, 012007

Scharmer, G. B., Bjelksjo, K., Korhonen, T. K., Lindberg, B., \& Petterson, B. 2003, Proc. SPIE, 4853, 341

Scharmer, G. B., Narayan, G., Hillberg, T., et al. 2008, ApJL, 689, L69

Sukhorukov, A. V., \& Leenaarts, J. 2016, arXiv:1606.05180

Tian, H., Xu, Z., He, J., \& Madsen, C. 2016, ApJ, 824, 96

Tortosa-Andreu, A., \& Moreno-Insertis, F. 2009, A\&A, 507, 949

Uitenbroek, H. 2001, ApJ, 557, 389

van Noort, M., Rouppe van der Voort, L., \& Löfdahl, M. G. 2005, SoPh, 228, 191

Vissers, G., \& Rouppe van der Voort, L. 2012, ApJ, 750, 22

Vissers, G. J. M., Rouppe van der Voort, L. H. M., \& Rutten, R. J. 2013, ApJ, 774,32

Vissers, G. J. M., Rouppe van der Voort, L. H. M., Rutten, R. J., Carlsson, M., \& De Pontieu, B. 2015, ApJ, 812, 11

Watanabe, H., Vissers, G., Kitai, R., Rouppe van der Voort, L., \& Rutten, R. J. 2011, ApJ, 736, 71

Xu, X.-Y., Fang, C., Ding, M.-D., \& Gao, D.-H. 2011, RAA, 11, 225 